\title{
CHARACTERISATION AND PERFORMANCE OF THE CERN ECR4 ION SOURCE
}

\author{
C. Andresen, J. Chamings, V. Coco, C.E. Hill, D. Kuchler, A. Lombardi, \\ E. Sargsyan and R. Scrivens
}

\begin{abstract}
To optimise the heavy ion injector for the LHC, a good knowledge of the parameters of the ECR4 ion source and the beam transport in the Low Energy Beam Transport (LEBT) for a lead ion beam is necessary. Results of the emittance measurements of the full beam $(\mathrm{O}+\mathrm{Pb})$ leaving the source using a scanning slit and profile monitor will be presented. Furthermore, the emittance of a single charge state after the source has been measured using a solenoid scan coupled to a guillotine and spectrometer. The results for last year's operation for the SPS fixed target physics with indium will also be presented.
\end{abstract}




\title{
Characterisation And Performance Of The CERN ECR4 Ion Source
}

\author{
C. Andresen, J. Chamings, V. Coco, C.E. Hill, D. Kuchler, A. Lombardi, \\ E. Sargsyan and R. Scrivens
}

AB Department, CERN, 1211 Geneva 23, Switzerland

\begin{abstract}
To optimise the heavy ion injector for the LHC, a good knowledge of the parameters of the ECR4 ion source and the beam transport in the Low Energy Beam Transport (LEBT) for a lead ion beam is necessary. Results of the emittance measurements of the full beam $(\mathrm{O}+\mathrm{Pb})$ leaving the source using a scanning slit and profile monitor will be presented. Furthermore, the emittance of a single charge state after the source has been measured using a solenoid scan coupled to a guillotine and spectrometer. The results for last year's operation for the SPS fixed target physics with indium will also be presented.
\end{abstract}

\section{INTRODUCTION}

Following the success of the light ion fixed target programme at the SPS, an international collaboration was formed to build a heavy ion injector [1] for the CERN complex and most especially for the SPS. This installation became operational in 1994 [2] providing Lead ions to physics. The programme came to an end in 2003 with a final operational period using Indium ions instead of the more usually requested Lead ions.

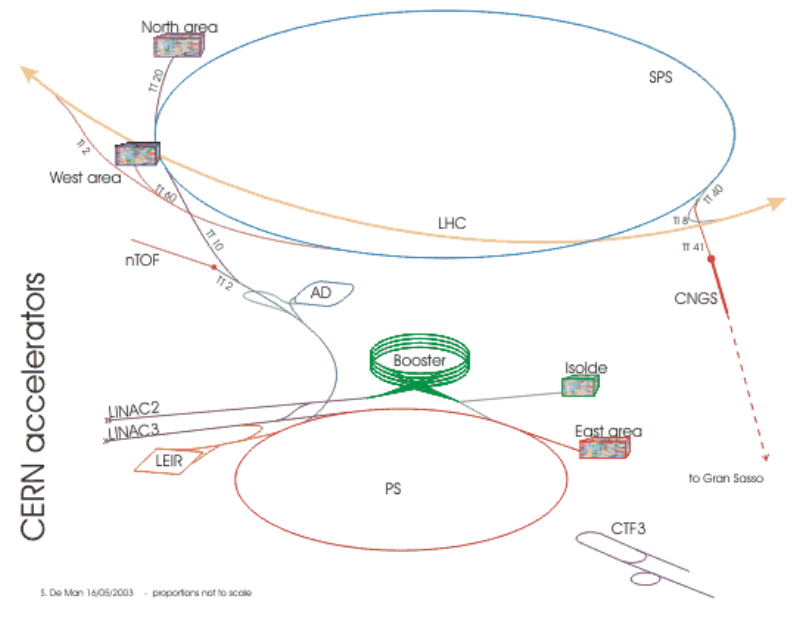

FIGURE 1. The CERN Accelerator Complex

Within the CERN accelerator complex (Fig. 1) the current major accelerator project is the Large Hadron
Collider (LHC) which is being constructed in the border zones between France and Switzerland to collide $2 * 7 \mathrm{TeV}$ proton beams deep underground [4]. Although the emphasis for the project has been on protons, Lead ion beams at $2.76 \mathrm{TeV} / \mathrm{u}$ have been requested by the physics community. To prepare for this new operating regime a programme of measurements on the ion beam were made to see if there were any fundamental limitations in the linac.

\section{IONS FOR LHC}

Using the present system of multiturn injection of the ions into the PS Booster (PSB), there would be a factor of 30 missing in beam brightness. This could be overcome by increasing the source current, but this would result in an unacceptable emittance growth in the beam which would be incompatible with the very tight emittance budget of the LHC injection chain. In view of CERN's considerable experience in phase space cooling, experiments demonstrated that it would be possible to stack and phase space cool heavy ions in a storage ring. By this means it proved possible to accumulate at least $25 \%$ of the required number of ions in the ring [4] with the linac running at $2.5 \mathrm{~Hz}$ instead of $0.8 \mathrm{~Hz}$. The stacking was limited by the balance between recombination losses due to the vacuum perturbations induced by beam losses and the injected beam. Thus by doubling the linac ion current 
and doubling the repetition rate, the goal could be achieved.

The majority of the components of the linac had been designed for $10 \mathrm{~Hz}$ operation so the repetition rate increase would only require the replacement of a few power supplies. The current increase was more problematic within the funding made available. It was felt that an increase in microwave frequency at the source from 14 to $18 \mathrm{GHz}$, the acceleration of $\mathrm{Pb}^{25+}$, relaxing the beam stability as the source could be tuned at each programmed LHC filling together with finding and compensating aperture restrictions, the equivalent of doubling the current could be achieved. Thus a campaign of beam measurements was started.

\section{SOURCE EMITTANCE}

During the design phase of Linac3 the emittance of the beam from the source in the pulsed afterglow mode was assumed to be the same as that as in d.c. operation. Inadequate time resolution of the measuring equipment and a lack of time during source commissioning meant that this was never remeasured. Measurements on the GANIL test bench which included a solenoid after the source indicated 100\% emittances of $<120 \mathrm{~mm}$.mrad at a waist [1].

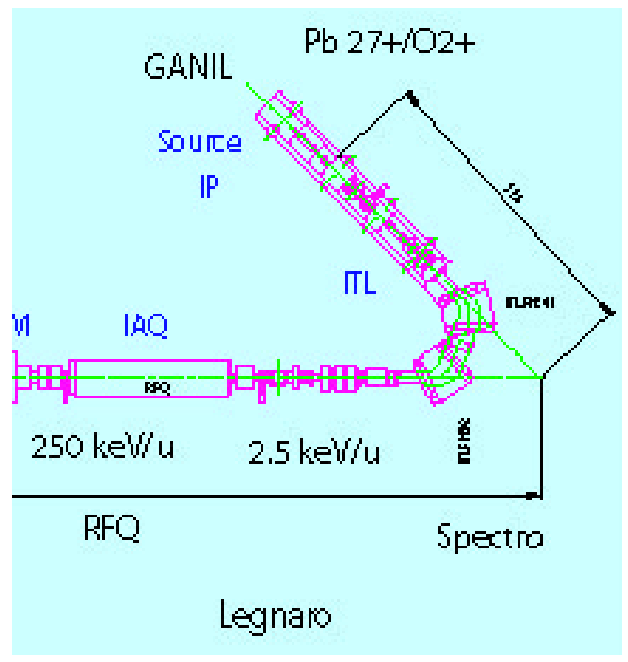

FIGURE 2. Low energy end of the ion linac, Linac3.

Following the end of physics operation, opportunity was taken to install a slit/collector emittance meter $600 \mathrm{~mm}$ downstream of the source extraction to investigate the source emittance. Fig. 2 shows the low energy end of the linac and the meter was installed in place of the solenoid after the source. This device had a small integration time (400 $\mu$ s) with acquisition at a user defined instant in time. The source was felt to be sufficiently stable that emittances did not change during the measurement or during the day . These measurements of the total $(\mathrm{O}+\mathrm{Pb})$ [5] beam extracted from the source on a typical operational afterglow beam indicated a 90\% geometrical (1 rms) during the afterglow of :- H 142 mm.mrad (22.0), V 192 mm.mrad (29.8). Fig. 3 is a overlay of the fitted 4 rms ellipses for 8 measurements in both planes which demonstrates the stability of the source and its reproducibility.

Of major interest was the evolution of the emittance during the afterglow Fig. 4 shows the evolution with time in the horizontal plane over the typical beam length used by the accelerator. The emittance can be seen to reduce by about $25 \%$ during the useful pulse. If a similar phenomenon were to be exhibited in the vertical plane, then there would be a substantial increase in beam brightness. However there was no evidence of an increase in beam intensity out of the linac during the beam pulse which would seem to indicate that there are no major acceptance limitations along the machine.

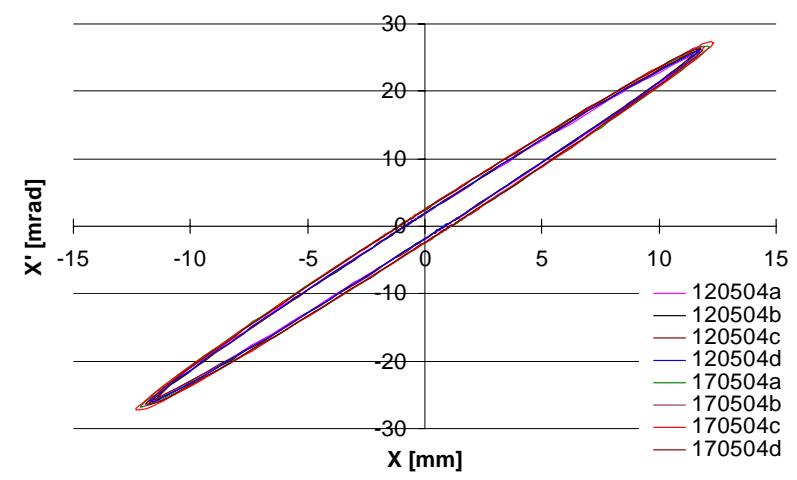

FIGURE 3. 4 rms emittances of 8 measurement (12xx H plane, 17xx V plane)

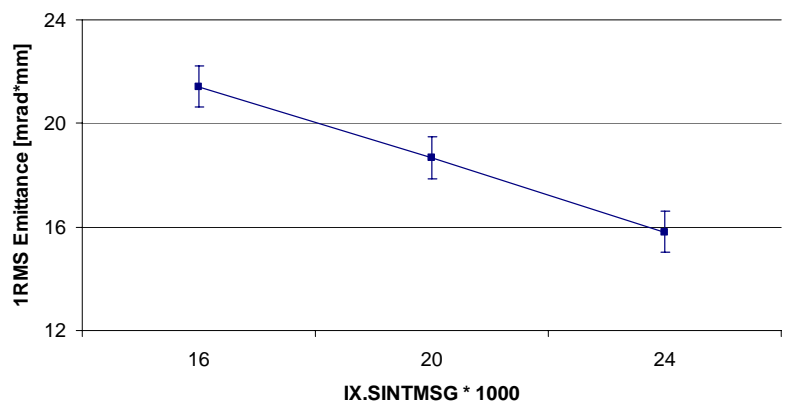

FIGURE 4. Evolution of horizontal emittance during the afterglow. The initial point is at the start of the afterglow. The last $800 \mu$ s later. 20 corresponds to PSB beam injection. 
Normal operation of the source used an afterglow that maximised both stability and intensity over the injectable part of the beam. Alternate settings could give a very smooth afterglow but at reduced intensity. The rms H,V emittances measured for an operational beam were typically $20.8 \mathrm{~mm}$.mrad, $35.6 \mathrm{~mm}$.mrad whereas for a smooth beam these became $16.9 \mathrm{~mm}$.mrad, $22.3 \mathrm{~mm}$.mrad. The operational beam was adjusted to maximise beam out of the stripper.

The density distribution of the particles in the beam has long been a subject of conjecture and screens in the PSB injection line gave some evidence that the beam, at the end of the linac, was of a strange form. A further observation with a slightly more sensitive screen attached to a parasitic experiment seemed to confirm this view. The images suggested that the beam had a triangular form with three hot spots in the corners of the triangle. The most probable source of this form would be the plasma density distribution due to the hexapolar field in the plasma in the extraction area. Figure 5 is an emittance plot of a typical beam showing the ion density distribution. The beam was observed to consist, typically, of ten separated islands of varying density whose position remained constant over a series of measurements but changed position as source parameters changed. This might suggest that the non uniform distribution already exists at the source exit. Staining and surface erosion on the puller electrode supports this conjecture. However, there is no scintillator screen at this point to verify this possibility

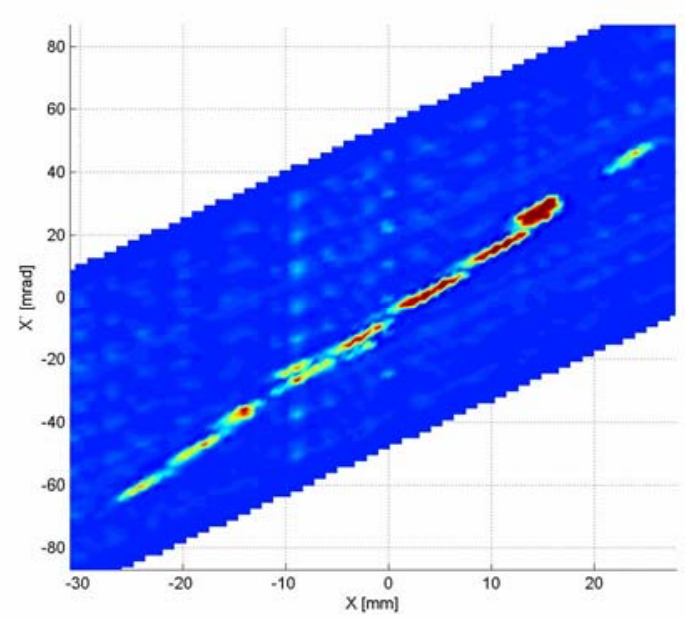

FIGURE 4. Emittance plot showing density islands.

\section{ION BEAM EMITTANCE}

A set of slits exists in a diagnostic box just upstream of the first spectrometer magnet and a Faraday cup downstream of the second magnet. Opportunity was taken to use this slit collector system in conjunction with a Charge State Distribution program and solenoid current scanning to measure the width of the beam [6]. Using Trace validated Mathcad routines, the emittance of a single charge state could be computed from this information. These measurements gave a geometrical emittance of $100 \mathrm{~mm} . \mathrm{mrad}$ for $\mathrm{Pb}^{27+}$ and $120 \mathrm{~mm}$.mrad for $\mathrm{In}^{21+}$ at the source extraction in the horizontal plane This technique which does not require any additional installation in the existing beam line should prove invaluable later when the linac is upgraded for LHC operation.

\section{INDIUM BEAMS}

Physics requested an intermediate mass ion beam for their last period of ion physics. The element selected would need to be mono-isotopic as the mass differences between isotopes would give capture difficulties in the following accelerator, thus wasting ions, and should have melting and vapour pressure qualities similar to Lead. For these reasons Indium was chosen.

Initial tests showed that the optimum charge state ${ }_{600}$ from the ECRIS was $\mathrm{In}^{21+}$ which resulted in an extraction voltage of approximately $15 \mathrm{kV}$ to meet the soo injection energy requirement for the RFQ of $2.5 \mathrm{keV} / \mathrm{u}$. This ion was stripped to $\mathrm{In}^{37+}$ after the ${ }^{00}$ linac. Typically 70-90 e $\mu \mathrm{A}$ of In $^{21+}$ was injected, after mass analysis and matching, into the RFQ

\section{PROBLEMS}

To obtain a vapour pressure of around $10^{-5} \mathrm{mbar}$ in the plasma chamber, the indium sample must be heated to around $650{ }^{\circ} \mathrm{C}$. Unfortunately, molten indium is capable of wetting many glasses and ceramics, a phenomenon accentuated by the vacuum environment inside the source. This wetting action caused the formation of short circuits inside the microoven used to evaporate the sample which resulted in considerable instabilities in the beam. Indium oxide charges were tried but the sample lifetime proved too short to be useful ( $<24$ hours). 
A solution to the problem was found in regulating the power delivered to the oven. For a given operating temperature the power needed to maintain this temperature is essentially constant. Variations may occur as emissivity changes with time but this is a slow process. Thus to a first order, the oven temperature should be constant for a fixed heating power. For lead operation, constant voltage control of the oven heating was sufficient to maintain source stability. For Indium this was not sufficient due to the varying impedance of the oven and thus a power controller was developed [7]. Once this had been installed the beam the beam intensity was generally reproducible to $\pm 10 \%$ (as compared to $\pm 5 \%$ for lead).

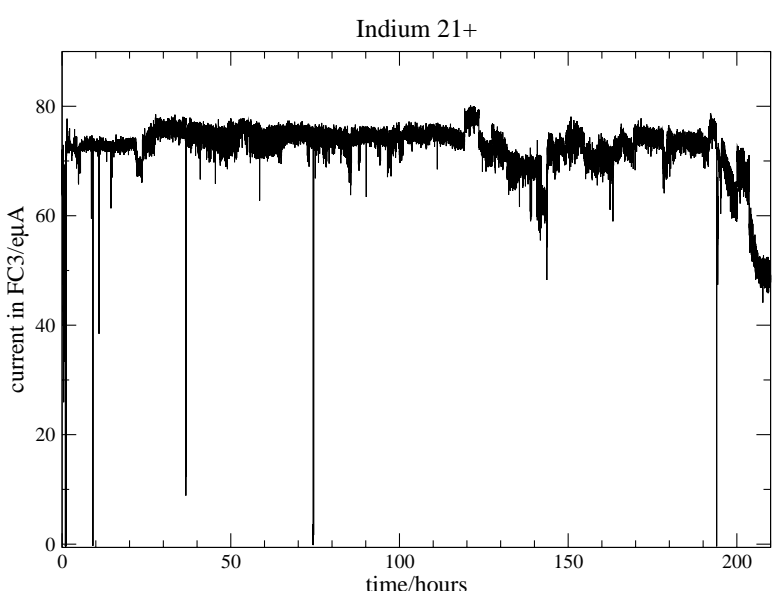

FIGURE 2. Typical long term stability of the Indium beam after the RFQ

Running the oven at a higher temperature resulted in a much reduced lifetime of the oven filament. Power control helped in this respect but tests showed that an operational life of $8 \pm 4$ days could be expected.

\section{PERFORMANCE}

The Indium beam was scheduled for a total run time of 1141 hours continuous. Of this time 28 hours were lost due to machine faults and another 28 for oven replenishments. Opportunity was taken from the presence of weekly LHC proton beam study sessions on the SPS to change the oven and sample at these times. This also ensured the presence of qualified personnel for the operation. Over the period in question the ion beam was maintained around 80 e $\mu \mathrm{A}$ which was more than sufficient to satisfy the requirements of physics [8].

\section{REFERENCES}

1. D. Warner, Editor. "CERN Heavy-Ion Facility Design Report”, CERN 93-01, 1993.

2. H. Charmot et al. "Operational Experience with the CERN Hadron Linacs", Proc. $18^{\text {th }}$ Int. Linac Conf., Geneva, 1996, CERN 96-07, 360, 1996.

3. O. Brüning et al .(Eds) "LHC Design Report, Vol 1, The Main Ring”, CERN 2004-003, 2004.

4. J. Bosser et al. "Experimental Investigations of Electron Cooling and Stacking of Lead Ions in a Low Energy Accumulation Ring” Particle Accelerators, 63, 171, 1999.

5. C. Andresen et al., "Measurement of the ECR4 Ions Source Emittance”, CERN AB-ABP, to be published.

6. J. Chamings, "Measurements on $\mathrm{Pb}^{27+}$ Sources for the CERN Heavy Ion Injection Chain”, M. Phys. Thesis, University of Surrey, GB (AB-Note-2004-44 (ABP), 2004).

7. C.E. Hill, D. Kuchler, M. O’Neil, "New Beam for the CERN Fixed Target Heavy Ion Programme”, Proc. $15^{\text {th }}$ Int. Workshop on ECR Sources, Jyväskylä, 2002, JYFL Research Report 4/2002, 2002.

8. J. Chamings et al., "New Operational Beam for the CERN Heavy Ion Programme", Proc. $10^{\text {th }}$ Int., Conf. Ion Sources, Dubna, 2003, Rev. Sci. Inst., 75,. 2004. 